\title{
Modeling and Measurement of Phase Noise in GaAs HBT Ka-Band Oscillators
}

\author{
Friedrich Lenk, Matthias Schott, and Wolfgang Heinrich \\ Ferdinand-Braun-Institut für Höchstfrequenztechnik (FBH), Albert-Einstein-Str. 11, \\ D-12489 Berlin / Germany, Tel.: +49-30-6392-2625, Fax: +49-30-6392-2642, \\ email: f.lenk@ieee.org,w.heinrich@ieee.org
}

\begin{abstract}
Accurate oscillator phase-noise simulation is a key problem in MMIC design, which is not solved satisfactory so far and needs further investigation. In this paper, a Ka-band MMIC oscillator with GalnP/GaAs HBT and on-chip resonator is treated as an example. Measured phase noise reaches $-90 \mathrm{dBc} / \mathrm{Hz}$ and below at $100 \mathrm{kHz}$ offset. To evaluate phase-noise prediction, the circuit is simulated using different commercial simulation tools and HBT models. Considerable differences in simulation results are observed.
\end{abstract}

\section{Motivation}

Low phase-noise oscillators are key components in communication as well as sensor systems for the emerging markets in the $20 \mathrm{GHz}$ to $80 \mathrm{GHz}$ range. In order to meet the low-cost requirements, monolithically integrated solutions (MMICs) with on-chip resonators are highly favorable. This demands for both transistors with low 1/f-noise and accurate CAD tools for circuit optimization. Regarding the transistor, the GalnP/GaAs HBT is particularly suited since its $1 / f-$ noise is considerably lower than for GaAs HEMTs, but the frequency potential is higher than that of SiGe HBTs. Phase-noise prediction, on the other hand, involves too much uncertainties so far to be a reliable basis for circuit design. Further investigations are required on this subject.

The purpose of our paper is to contribute results in this field. As an example, we study a GaAs-HBT-based $36 \mathrm{GHz}$ oscillator. Attention focuses on the phase noise at $100 \mathrm{kHz}$ offsetfrequency. From the measurements, it can be seen that this frequency is in the transition region between $1 / f^{3}$ and $1 / f^{2}$ phase-noise behavior. Phase-noise modeling, therefore, needs to be composed of the following issues [6]:

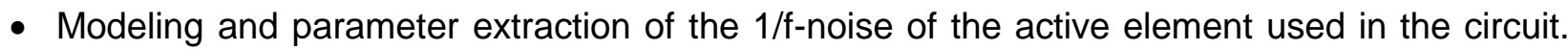
Measuring 1/f-noise of the GalnP/GaAs-HBTs at different source impedances and bias states, we found two independent low-frequency (LF) noise sources. The two noise sources are implemented in an in-house HBT large-signal model. The results are compared to simulations with a standard Gummel-Poon model, which includes only a single LF noise source.

- Calculation of the conversion from LF noise into oscillator phase-noise. The simulations of the $36 \mathrm{GHz}$ oscillator are performed using 3 different commercial design tools (MDS and ADS of Agilent and Serenade of ANSOFT).

\section{1/f-Noise HBT Description}

In standard HBT models (e.g. Gummel-Poon) only the base current includes 1/f-noise. However, measurements of the spectral current density of $1 / \mathrm{f}$-noise at the output of $\mathrm{GalnP} / \mathrm{GaAs}$ HBTs with different source resistances $R_{s}$ show that this description is not sufficient. In the lowfrequency range, all of the extrinsic reactive elements can be neglected. Hence, the equivalent circuit of the HBT simplifies to a $Y$ matrix with $R_{\text {be }}$, and $\beta$ and an additional $Z$ matrix containing the parasitic resistors $R_{b}, R_{c}$, and $R_{e}$ (see Fig. 1) The 1/f-noise model should be consistent with the high-frequency noise model, so we suppose voltage sources at the extrinsic resistors and current sources at base and collector current.

Using the simplified equivalent circuit, the influence of each noise source on output noise can be calculated as a function of the source resistance. In general, measurements for $n$ different source-resistance values allow the separation of up to $n$ independent noise sources, but for the simplified HBT equivalent circuit the voltage sources at base and emitter cannot be separated. From the simulation point of view, both contribute the same noise at the output, but from physi- 
cal considerations one can conclude that the emitter layer acts as the relevant LF noise source [1]. In Fig. 3, measured LF noise at the output is plotted together with the modeled data. All bias and source resistance conditions are well described.

\section{Circuit Simulation and MMIC Fabrication}

The oscillator is designed as a negative resistance circuit in coplanar environment. Fig. 2 provides a chip photo. Lumped elements are used as blocking capacitors only. The resonator is formed by a short-circuited line at the emitter.

All coplanar line elements are simulated with the model according to [2]. For HBT description the in-house model [3] extended by two $1 / \mathrm{f}$-noise sources is employed. For the original design, MDS of Agilent was applied using both the in-house HBT model [3] and the built-in GummelPoon one. Fig. 4 presents the results together with measured phase noise. Both models fail to predict phase noise correctly. The in-house model yields reasonable agreement at $100 \mathrm{kHz}$ offset, but frequency dependence does not fit.

The circuits are fabricated using the 4 inch GaAs-HBT MMIC process at the FBH. For details see $[4,5]$. To suppress recombination at the surface of the GaAs-base layer, a fully depleted GalnP ledge technology is applied. The electroplated Au metalization is $3 \mu \mathrm{m}$ thick, i.e., the process does not involve special measures to improve $Q$ factor of the passive elements.

\section{Phase-Noise Simulation and Measurements}

To evaluate $\mathrm{mm}$-wave phase-noise prediction, the circuit is simulated employing three different commercial design tools: MDS and ADS of Agilent and Serenade of Ansoft. Furthermore, the large-signal HBT model as well as the CPW model is varied applying the built-in models and the user-defined CPW [2] and HBT model [3], respectively. Tab. 1 provides the results.

The first observation is the large spread in the simulation results, mainly with regard to output power and phase noise. Using the in-house CPW model instead of the ADS built-in one, for example, does not influence output power and frequency greatly, as expected, but leads to more than $17 \mathrm{~dB}$ deviation in phase noise. Similarly, comparing the ADS and MDS data for identical in-house models, only phase-noise results vary, though by not less than $30 \mathrm{~dB}$. For the GP model, on the other hand, both power and phase noise changes considerably. Given the deviations in power, i.e., in the noiseless nonlinear analysis, it is not surprising that the spread in phase-noise data is even larger. In this regard, the influence of the HBT large-signal model should be emphasized. Difficult to explain, however, are the differences between the three simulators for otherwise identical models.

\section{Conclusions}

Ka-band MMIC oscillators based on GalnP/GaAs HBTs yield excellent phase noise values exceeding $-90 \mathrm{dBc} / \mathrm{Hz}$ at $100 \mathrm{kHz}$ offset. Large-signal and phase noise simulations using the common design tools, however, fail to predict this and show an unexpectedly large spread in data. Even an advanced 1/f-noise description of the HBT does not lead to adequate results. This finding points out the high sensitivity of these quantities to details in transistor modeling and simulation approach.

\section{References}

[1] P. Heymann, M. Rudolph, R. Doerner, and F. Lenk "Modeling of Low-Frequency Noise in GalnP/GaAs Hetero-Bipolar Transistors," MTT-S Int. Microwave Symp. Dig, 2001, pp. 1967-1970

[2] W. Heinrich, "Quasi-TEM description of MMIC coplanar lines including conductor loss effects," IEEE Transactions on Microwave Theory and Techniques, Vol. 41, pp. 45-52, Jan. 1993.

[3] M. Rudolph, R. Doerner, K. Beilenhoff, P. Heymann, "Scalable GalnP/GaAs HBT largesignal model," 2000 Int. Microw. Symp. Dig., pp. 753-756 
[4] H. Kuhnert, F. Lenk, J. Hilsenbeck, J. Würfl, and W. Heinrich, "Low Phase-Noise GalnP/GaAs-HBT MMIC Oscillators up to $36 \mathrm{GHz}$," MTT-S Int. Microwave Symp. Dig, 2001, pp. 1551-1554

[5] M. Achouche, T. Spitzbart, P. Kurpas, F. Brunner, J. Würfl, G. Tränkle: "High Performance InGaP/GaAs HBTs for Mobile Communications," Electr. Letters, Vol. 36, No. 12, 2000, pp. 1073-1075.

[6] M. Regis et al ,"Non-Linear Noise Mechanisms in SiGe BiCMOS Devices," 2000 Topical Meeting on Silicon Monolithic Integrated Circuits in RF Systems, Digest, pp. 163-166.

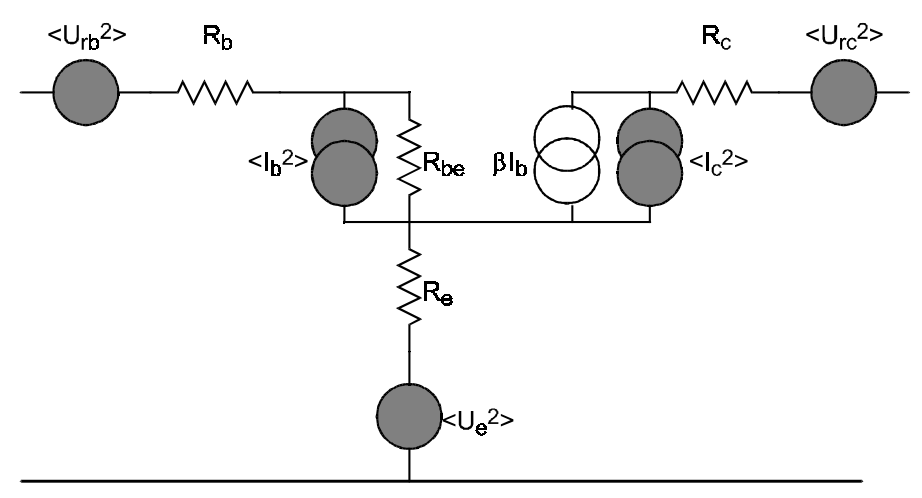

Fig. 1 Equivalent noise circuit of the HBT (reactive elements are neglected).

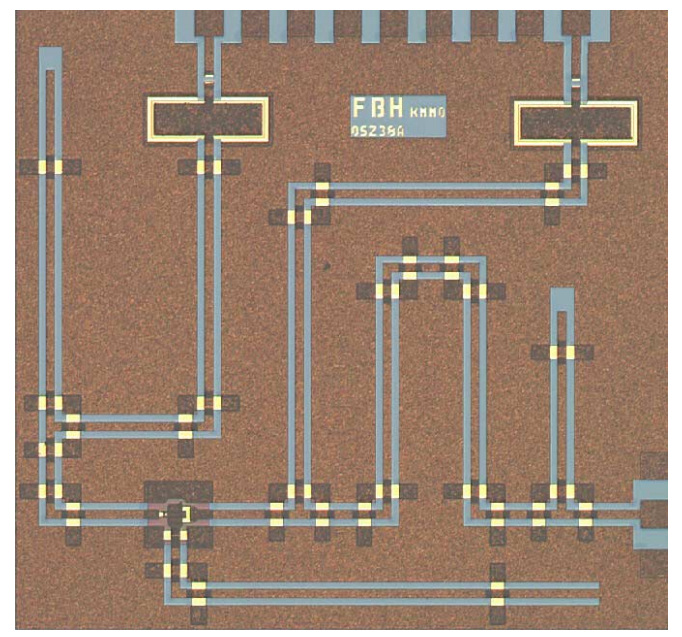

Fig. 2 Chip photo of the MMIC oscillator (size: $1.42 \times 1.34 \mu \mathrm{m}^{2}$ ). 


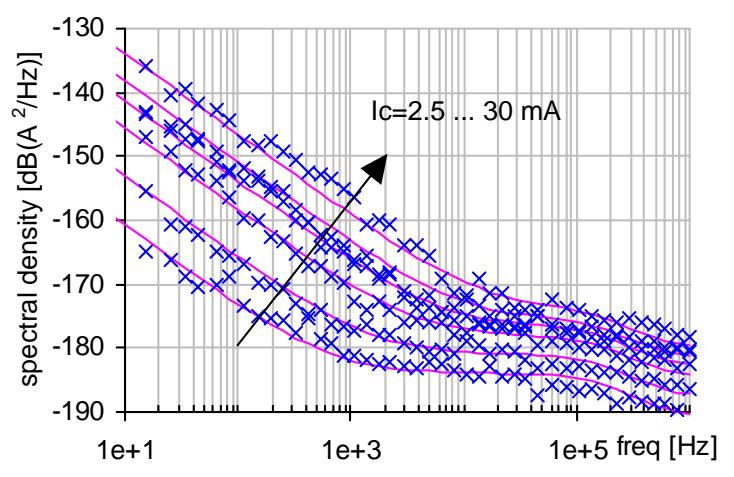

(a)

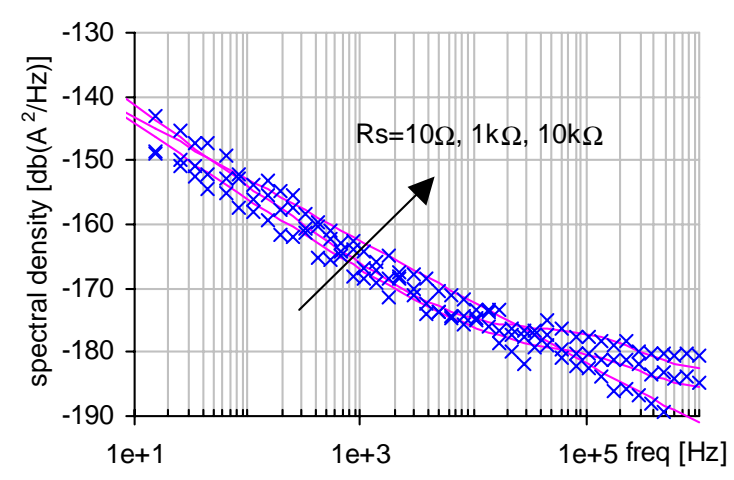

(b)

Fig. 3 Measured (symbols) and modeled (lines) LF current-noise in $\mathrm{dB}\left(\mathrm{A}^{2} / \mathrm{Hz}\right)$ vs. frequency at the output of a $1 \times 3 \times 30 \mu \mathrm{m}^{2} \mathrm{HBT}$; (a) input resistance $R_{s}=10 \mathrm{k} \Omega$ and collector current $\mathrm{I}_{\mathrm{c}}$ as parameter; (b) collector current $I_{c}=15 \mathrm{~mA}$ and source resistance $R_{s}$ as parameter.

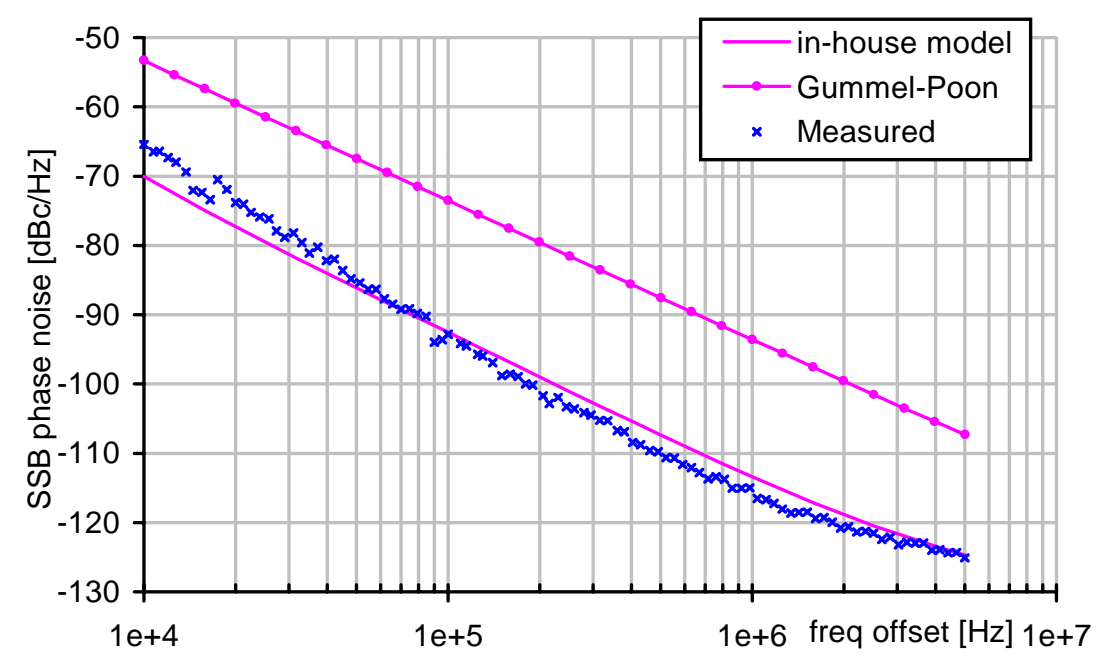

Fig. 4 Phase noise vs. frequency: measured (symbols) and modeled (lines) data using Gummel-Poon and in-house HBT model.

\begin{tabular}{|c|c|c|c|c|c|}
\hline CPW model & $\begin{array}{c}\text { HBT } \\
\text { model }\end{array}$ & $\begin{array}{c}\text { Simulation- } \\
\text { tool }\end{array}$ & $\begin{array}{c}\text { Output power } \\
{[\mathrm{dBm}]}\end{array}$ & $\begin{array}{c}\text { Frequency } \\
{[\mathrm{GHz}]}\end{array}$ & $\begin{array}{c}\text { Phase noise } \\
@ 100 \mathrm{kHz} \\
{[\mathrm{dBc} / \mathrm{Hz}]}\end{array}$ \\
\hline std.-CPW & in-house & ADS & 2.475 & 35.15 & -82.299 \\
\hline std.-CPW & GP & ADS & 10.532 & 35.76 & -89.333 \\
\hline in-house & in-house & ADS & 2.435 & 35.05 & -63.349 \\
\hline in-house & GP & ADS & 10.91 & 35.82 & -72.090 \\
\hline in-house & in-house & MDS & 2.242 & 34.90 & -92.540 \\
\hline in-house & GP & MDS & 2.610 & 35.79 & -82.541 \\
\hline in-house & GP & SERENADE & 8.280 & 35.80 & -82.950 \\
\hline \multicolumn{6}{|c|}{ measurements } \\
\hline
\end{tabular}

Tab. 1 Simulation results for Ka-band oscillator. 